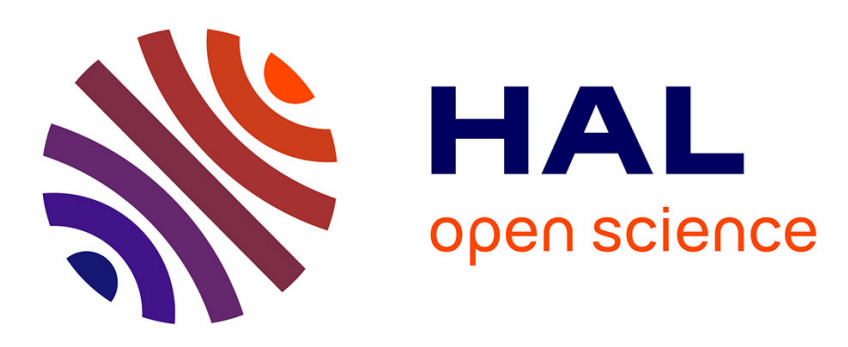

\title{
Linearized behavior of a dissipative rubber with large static preloads
}

\author{
Stéphane Lejeunes, Thien An Nguyen Van, Adnane Boukamel, Dominique \\ Eyheramendy
}

\section{- To cite this version:}

Stéphane Lejeunes, Thien An Nguyen Van, Adnane Boukamel, Dominique Eyheramendy. Linearized behavior of a dissipative rubber with large static preloads. Computers \& Structures, 2012, 96-97, pp.46-53. 10.1016/j.compstruc.2012.01.006 . hal-00676317

\section{HAL Id: hal-00676317 https://hal.science/hal-00676317}

Submitted on 11 Jan 2018

HAL is a multi-disciplinary open access archive for the deposit and dissemination of scientific research documents, whether they are published or not. The documents may come from teaching and research institutions in France or abroad, or from public or private research centers.
L'archive ouverte pluridisciplinaire HAL, est destinée au dépôt et à la diffusion de documents scientifiques de niveau recherche, publiés ou non, émanant des établissements d'enseignement et de recherche français ou étrangers, des laboratoires publics ou privés. 


\title{
Linearized behavior of a dissipative rubber with large static preloads
}

\author{
S. Lejeunes ${ }^{*, a}$, T.A. Nguyen Van ${ }^{\mathrm{a}}$, A. Boukamel ${ }^{\mathrm{b}}$, D. Eyheramendy ${ }^{\mathrm{a}, \mathrm{c}}$ \\ ${ }^{a}$ LMA, CNRS, UPR7051, Aix-Marseille Univ, F-13402 Marseille Cedex 20, France \\ ${ }^{b}$ EHTP, KM 7, Route d'El Jadida, B.P 8108, Oasis, Casablanca, Maroc \\ ${ }^{c}$ Centrale Marseille, F-13451 Marseille Cedex 20, France
}

\begin{abstract}
The dynamic behavior of filled rubber has been intensively studied during the last 20 years and a large number of finite strain models have been developed. However, many industrial applications involve small vibrations superimposed on large static preloads. In this specific case, the dynamic problem can be treated as a small amplitude problem near a finite strain state. In this paper, we discuss how to extend previous approaches by taking arbitrary (large) dynamic rotations into account. A general approach is proposed as well as a finite element implementation. Results show that large rotations are required in some tests, even at small dynamic amplitudes.
\end{abstract}

Key words: finite strain, viscoelasticity, linearization

\section{Introduction}

In the field of industrial anti-vibratory systems, rubber-based solutions are often preferred to hydrodynamic ones. These parts are submitted to multi-axial loadings with both static and dynamic contributions. For instance in helicopters the power transmission can be isolated from the cab by the help of sandwich rubber mountings. In this application, rubber mountings are submitted to shear vibrations superimposed on a large static compression load. As the objective is to dissipate energy, these parts are made of filled rubber. The fillers may consist in carbon black or silica and the elastomeric matrix can be made of synthetic or natural rubber, depending on the mechanical characteristics required. This complex composite material undergoes some specific effects, which result directly from the loading history, such as the Mullins effect, Payne effect and Fletcher-Gent effect (see [1, 2, 3] and references therein). Typically, in the context of small harmonic loading superimposed on an initially static finite strain state, filled rubbers exhibit a dependency of the storage modulus and the loss modulus upon static preload. It therefore seems quite natural to adopt a linearized formulation to account for small vibrations under finite strain conditions and the non-linear effects of the preloading.

This topic has been discussed in the literature since the study by Lianis [4], who developed a theory of small viscoelastic motion superimposed on large static deformations. Morman \& Nagtegaal developed a general formulation in a Eulerian framework, focusing on harmonic motions, and presented a finite element implementation in the frequency space [5]. A similar strategy was applied by Kim et al. [6, 7], who consistently linearized a finite strain viscoelastic model and presented a finite element implementation of their model. Höfer \& Lion recently developed a finite strain model accounting for the Payne effect in the time domain, for modeling transient behavior [8]. A linearized version of the latter model under large static preloading loads was proposed, which enabled the authors to express the storage and loss modulus analytically (see [9]). The latter studies also give some interesting experimental results reflecting the dynamic behavior of rubber at small strain amplitudes, but other studies can also be consulted in this context [10, 11].

\footnotetext{
${ }^{*}$ Corresponding author

Email addresses: lejeunes@lma.cnrs-mrs.fr, tel: $\quad 033491054382$, fax: 033491054749 (S. Lejeunes), thienandlma.cnrs-mrs.fr (T.A. Nguyen Van), boukameleehtp.ac.ma (A. Boukamel), dominique.eyheramendy@centrale-marseille.fr (D. Eyheramendy)

Preprint submitted to Elsevier 
In this paper, we propose an original linearization framework that can take into account material rotations. The main point of this approach lies in the multiplicative decomposition of the transformation gradient into static and dynamic parts and the polar decomposition of the dynamic one. A small dynamic strain tensor is defined from the pure dynamic deformation and the stress linearization is derived. We show that the hypothesis of an additive splitting of the stress, which has often been used in previous papers, is not applicable in the context of large rotations. The present approach is general and can be applied independently of the constitutive model chosen to represent viscoelasticity. For more complex behavior exhibiting plasticity, a special attention must be paid as the static part may not be elastic. In this case additional hypothesis are necessary to derive the stress linearization. In this paper, we adopt a Zener model. The model linearization and the finite element implementation are presented.

In the first section, the thermodynamic principles and the finite strain rheological model are briefly presented. Given the variational formulation adopted, the choice of an appropriate configuration for the linearization procedure is discussed in the second section. The linearization strategy and the numerical implementation are addressed in the third section. In the last section, some numerical examples allows us to evaluate the model.

\section{Constitutive modeling}

\subsection{Thermodynamic basis}

In the context of the thermodynamics of irreversible processes, the constitutive equations must fulfill the Clausius Duhem inequality, which takes the following form in the Eulerian configuration and in the isothermal and adiabatic case:

$$
\mathcal{D}_{\text {int }}=\boldsymbol{\sigma}: \mathbf{D}-J^{-1} \rho_{0} \dot{\psi} \geq 0
$$

where $\boldsymbol{\sigma}$ is the Cauchy stress, $\mathbf{D}$ is the Eulerian rate of deformation, $J=\operatorname{det} \mathbf{F}$ is the volume variation (and $\mathbf{F}$ is the deformation gradient), $\rho_{0}$ is the volumetric mass in the initial configuration, $\mathcal{D}_{\text {int }}$ is the intrinsic dissipation and $\psi$ is the specific free energy.

As described in [12], the deformation gradient is first split into its volumetric and isochoric parts. The isochoric part is then split into and elastic and an inelastic parts, as shown in figure 1:

$$
\mathbf{F}=\left(J^{\frac{1}{3}} \mathbf{1}\right) \cdot \overline{\mathbf{F}}=\left(J^{\frac{1}{3}} \mathbf{1}\right) \cdot \overline{\mathbf{F}}_{\mathbf{e}} \cdot \overline{\mathbf{F}}_{\mathbf{v}}
$$

where $\overline{\mathbf{F}}$ is the incompressible part of the deformation gradient, $\overline{\mathbf{F}}_{\mathbf{e}}$ is an incompressible elastic deformation gradient and $\overline{\mathbf{F}}_{\mathbf{v}}$ is an incompressible inelastic deformation gradient accounting for the viscosity. These choices mean that the non-elastic processes involved will be purely isochoric and all the volume-changing deformations are assumed to be reversible. The free energy is assumed to be a function of the left Cauchy-Green tensor $\left(\overline{\mathbf{B}}=\overline{\mathbf{F}} \cdot \overline{\mathbf{F}}^{\mathrm{T}}\right)$, the left elastic Cauchy-Green tensor $\left(\overline{\mathbf{B}}_{\mathbf{e}}=\overline{\mathbf{F}}_{\mathbf{e}} \cdot \overline{\mathbf{F}}_{\mathbf{e}}^{\mathrm{T}}\right)$ and $J$. The time derivative of the free energy is therefore:

$$
\dot{\psi}=\frac{\partial \psi}{\partial \overline{\mathbf{B}}}: \dot{\overline{\mathbf{B}}}+\frac{\partial \psi}{\partial \overline{\mathbf{B}}_{\mathbf{e}}}: \dot{\mathbf{B}}_{\mathbf{e}}+\frac{\partial \psi}{\partial J} \dot{J}
$$

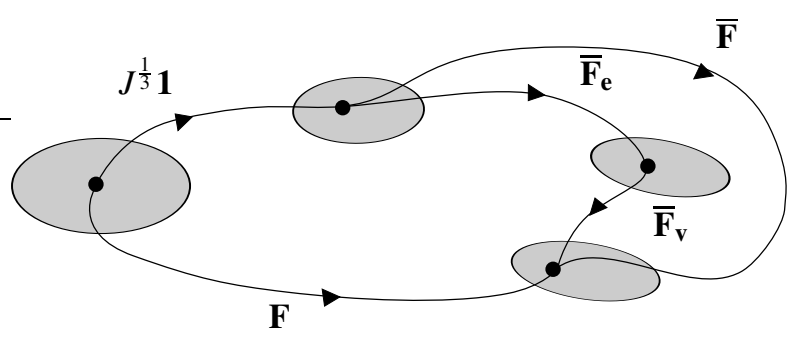

Figure 1: Splitting of the deformation gradient into an isochoric part, an elastic part and a viscous part. 


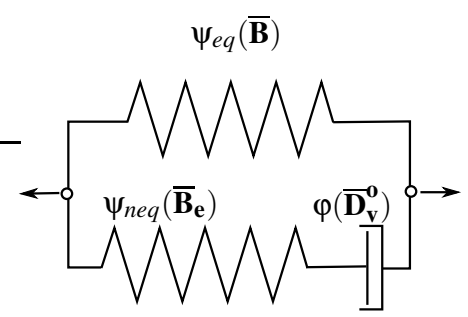

Figure 2: Simple model of finite strain viscosity: the Zener model.

where the time derivative of $J$ is defined by

$$
\dot{J}=J(\mathbf{1}: \mathbf{L}) \quad \text { with } \quad \mathbf{L}=\dot{\mathbf{F}} \mathbf{F}^{-1}
$$

and the time derivative of the strain variables is given by:

$$
\begin{aligned}
& \dot{\overline{\mathbf{B}}}=\mathbf{L} \cdot \overline{\mathbf{B}}+\overline{\mathbf{B}} \cdot \mathbf{L}^{\mathrm{T}}-\frac{2}{3}(\mathbf{1}: \mathbf{D}) \overline{\mathbf{B}} \\
& \dot{\overline{\mathbf{B}}}_{\mathbf{e}}=\mathbf{L} \cdot \overline{\mathbf{B}}_{\mathbf{e}}+\overline{\mathbf{B}}_{\mathbf{e}} \cdot \mathbf{L}^{\mathrm{T}}-2 \overline{\mathbf{V}}_{\mathbf{e}} \cdot \overline{\mathbf{D}}_{\mathbf{v}}^{\mathbf{o}} \cdot \overline{\mathbf{V}}_{\mathbf{e}}-\frac{2}{3}(\mathbf{1}: \mathbf{D}) \overline{\mathbf{B}}_{\mathbf{e}}
\end{aligned}
$$

where $\overline{\mathbf{D}}_{\mathbf{v}}^{\mathbf{0}}$ is the objective Eulerian inelastic rate of deformation, defined by

$$
\overline{\mathbf{D}}_{\mathbf{v}}^{\mathbf{o}}=\mathbf{R}_{\mathbf{e}} \cdot \overline{\mathbf{D}}_{\mathbf{v}} \cdot \mathbf{R}_{\mathbf{e}}^{\mathrm{T}}
$$

where $\mathbf{R}_{\mathbf{e}}$ results from the polar decomposition $\overline{\mathbf{F}}_{\mathbf{e}}=\overline{\mathbf{V}}_{\mathbf{e}} \cdot \mathbf{R}_{\mathbf{e}}$ and $\overline{\mathbf{D}}_{\mathbf{v}}=\left(\dot{\mathbf{F}_{\mathbf{v}}} \cdot \overline{\mathbf{F}}_{\mathbf{v}}{ }^{-1}\right)_{\text {sym }}$.

\subsection{Finite strain modeling: the Zener model}

The Zener model is composed by a Maxwell viscoelastic element put in parallel with an elastic one (see figure 2). In the finite strain context, this rheological model can be obtained by taking the following expression for the free specific energy:

$$
\psi=\psi_{e q}(\overline{\mathbf{B}})+\psi_{\text {neq }}\left(\overline{\mathbf{B}}_{\mathbf{e}}\right)+\psi_{\text {vol }}(J)
$$

where $\psi_{e q}(\overline{\mathbf{B}})$ is an equilibriated free energy, resulting from the instantaneous elasticity, and $\psi_{\text {neq }}\left(\overline{\mathbf{B}}_{\mathbf{e}}\right)$ is a nonequilibriated one, depending on the time effects.

After substituting Eqs. (5),(6) and (4) into Eq. (1), the following expression for the intrinsic dissipation is obtained:

$$
\begin{aligned}
\mathcal{D}_{\text {int }}= & \left(\boldsymbol{\sigma}-\rho_{0} J^{-1}\left(2 \overline{\mathbf{B}} \cdot \frac{\partial \psi_{e q}}{\partial \overline{\mathbf{B}}}\right)^{\mathrm{D}}-\rho_{0} J^{-1}\left(2 \overline{\mathbf{B}}_{\mathbf{e}} \cdot \frac{\partial \psi_{\text {neq }}}{\partial \overline{\mathbf{B}}_{\mathbf{e}}}\right)^{\mathrm{D}}-\rho_{0}\left(\frac{\partial \psi_{v o l}}{\partial J} \mathbf{1}\right)\right): \mathbf{D} \\
& +\left(2 \rho_{0} J^{-1} \overline{\mathbf{V}}_{\mathbf{e}} \cdot \frac{\partial \psi_{\text {neq }}}{\partial \overline{\mathbf{B}}_{\mathbf{e}}} \cdot \overline{\mathbf{V}}_{\mathbf{e}}\right): \overline{\mathbf{D}}_{\mathbf{v}}^{\mathbf{o}} \geq 0
\end{aligned}
$$

where the superscript ${ }^{\mathrm{D}}$ stands for the deviatoric operator. For arbitrary choices of $\mathbf{D}$, the constitutive equations are obtained as well as a remainder inequality governing the non-negativeness of the internal dissipation. The stress is defined as the sum of a deviatoric equilibrium part, a deviatoric overstress part and a spherical one:

$$
\boldsymbol{\sigma}=\overbrace{\left(2 \rho_{0} J^{-1} \overline{\mathbf{B}} \cdot \frac{\partial \psi_{e q}}{\partial \overline{\mathbf{B}}}\right)^{\mathrm{D}}}^{\boldsymbol{\sigma}_{e q}}+\overbrace{\left(2 \rho_{0} J^{-1} \overline{\mathbf{B}}_{\mathbf{e}} \cdot \frac{\partial \psi_{\text {neq }}}{\partial \overline{\mathbf{B}}_{\mathbf{e}}}\right)^{\mathrm{D}}}^{\boldsymbol{\sigma}_{\text {neq }}}+\overbrace{\rho_{0} \frac{\partial \psi_{v o l}}{\partial J} \mathbf{1}}^{\boldsymbol{\sigma}_{v o l}}
$$


By introducing a pseudo-potential of dissipation $\varphi$, which is a convex positive function of the objective rate of the internal variable $\overline{\mathbf{D}}_{\mathbf{v}}^{\mathbf{0}}$ and by applying the normality principle (see $[13,14]$ ), the following complementary law is then obtained:

$$
\frac{\partial \varphi}{\partial \overline{\mathbf{D}}_{\mathbf{v}}^{\mathbf{0}}}=\left(2 \rho_{0} J^{-1} \overline{\mathbf{V}}_{\mathbf{e}} \cdot \frac{\partial \psi_{\text {neq }}}{\partial \overline{\mathbf{B}}_{\mathbf{e}}} \cdot \overline{\mathbf{V}}_{\mathbf{e}}\right)^{\mathrm{D}}
$$

In this paper, we chose the simplest form of the pseudo-potential of dissipation, i.e. a quadratic form:

$$
\varphi\left(\overline{\mathbf{D}}_{\mathbf{v}}^{\mathbf{o}}\right)=\frac{\eta}{2}\left(\overline{\mathbf{D}}_{\mathbf{v}}^{\mathbf{o}}: \overline{\mathbf{D}}_{\mathbf{v}}^{\mathbf{o}}\right)
$$

where $\eta$ is the viscosity parameter. Eq. (11) becomes

$$
\overline{\mathbf{D}}_{\mathbf{v}}^{\mathbf{o}}=\frac{2 \rho_{0}}{J \eta}\left(\overline{\mathbf{V}}_{\mathbf{e}} \cdot \frac{\partial \psi_{\text {neq }}}{\partial \overline{\mathbf{B}}_{\mathbf{e}}} \cdot \overline{\mathbf{V}}_{\mathbf{e}}\right)^{\mathrm{D}}
$$

By substituting Eq. (13) into (6) and using the isotropy ${ }^{1}$ properties of $\psi_{\text {neq }}$, the following evolution equation is obtained for the Zener model:

$$
\dot{\overline{\mathbf{B}}}_{\mathbf{e}}=\mathbf{L} \cdot \overline{\mathbf{B}}_{\mathbf{e}}+\overline{\mathbf{B}}_{\mathbf{e}} \cdot \mathbf{L}^{\mathrm{T}}-\frac{2}{3}(\mathbf{1}: \mathbf{L}) \overline{\mathbf{B}}_{\mathbf{e}}-\frac{2}{\eta} \boldsymbol{\sigma}_{n e q} \cdot \overline{\mathbf{B}}_{\mathbf{e}}
$$

\section{Variational formulation}

\subsection{Comments on the internal virtual work}

To obtain a consistent variational formulation, three different expressions for the internal virtual work can be used, depending on the configuration considered:

$$
\mathcal{P}_{\text {int }}=\int_{\Omega_{0}} \mathbf{S}: \delta \mathbf{E}=\int_{\Omega_{0}} \boldsymbol{\Pi}: \delta \mathbf{F}=\int_{\Omega} \boldsymbol{\sigma}: \delta \mathbf{D}
$$

where $\mathbf{S}$ and $\boldsymbol{\Pi}$ are the second and first Piola Kirchoff stress tensors, and $\Omega_{0}$ and $\Omega$ are the reference and current configurations. The variational tensors $\delta \mathbf{E}, \delta \mathbf{F}$ and $\delta \mathbf{D}$ are defined by:

$$
\begin{aligned}
\delta \mathbf{E} & =\frac{1}{2}\left(\nabla_{\mathbf{X}} \delta u+\nabla_{\mathbf{X}}^{\mathrm{T}} \delta u+\nabla_{\mathbf{X}}^{\mathrm{T}} \delta u \nabla_{\mathbf{X}} \delta u\right) \\
\delta \mathbf{F} & =\nabla_{\mathbf{X}} \delta u \\
\delta \mathbf{D} & =\left(\nabla_{\mathbf{X}} \delta u\right)_{s y m}
\end{aligned}
$$

where $\nabla_{\mathbf{X}}$ and $\nabla_{\mathbf{X}}$ are the gradient operators with respect to the reference and current configurations, and $\delta u$ is a virtual kinematic field. It can be seen from these equations that only $\delta \mathbf{F}$ is linear with respect to the virtual field. Since the linearization is conduced about a large static deformation, the choice of a configuration for the internal virtual work is not obvious. The variational formulation in this case has to include linear measures of stress and strain. A $\Pi, \delta \mathbf{F}$ formulation was adopted here as the gradient operator does not have to be changed between a large and small strain steps. However, as a Eulerian version of the Zener model was chosen in the previous section, the Cauchy stress has to be pulled back before the stress linearization.

\footnotetext{
${ }^{1}$ In this paper, only the case of isotropic materials is considered.
} 


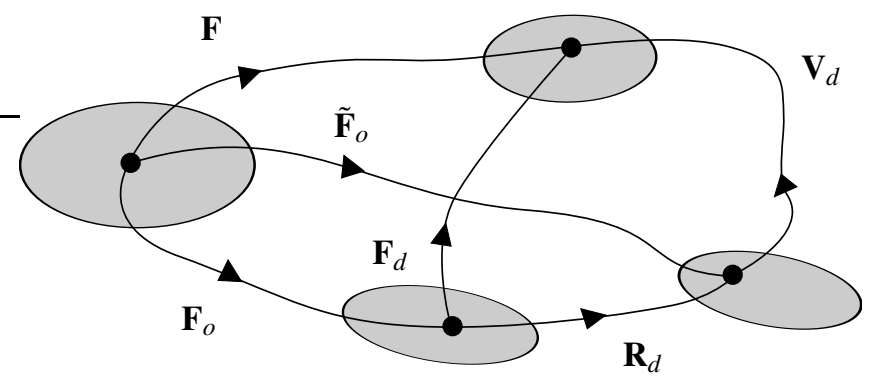

Figure 3: Intermediate states for the linearization of a dynamic transformation $\left(\mathbf{F}_{d}\right)$ with a static preloading $\left(\mathbf{F}_{o}\right)$.

\subsection{Nearly-incompressible media}

To account for the nearly-incompressible behavior of the material, we use a so-called perturbed Lagrangian variational form. In this mixed formulation, the Lagrangian parameter plays the role of a hydrostatic pressure, because of the decomposition of $\mathbf{F}$ into its isochoric and volumetric parts (see [15] for details). Introducing the pair (u; $p$ ) of kinematic and hydrostatic pressure, the solution of the equilibrium problem has to cancel the following integral form for all the test functions $\delta \mathbf{u}$ and $\delta p$ chosen in the same spaces as those of the trial functions $\mathbf{u}$ and $p$ respectively ( $\mathbf{f}_{v o l}$ and $\mathbf{f}_{\text {surf }}$ are the volumetric external forces and the surface ones, respectively):

$$
\left\{\begin{array}{l}
\int_{\Omega_{0}} \Pi: \nabla \delta u d \Omega-\int_{\Omega_{0}} \delta u \cdot \mathbf{f}_{\mathbf{v o l}} d \Omega-\int_{\delta \Omega_{0}} \delta u \cdot \mathbf{f}_{\text {surf }} d S \\
\int_{\Omega_{0}}\left(\rho_{0} \frac{\partial \psi_{v o l}}{\partial J}-p\right) \delta p d \Omega
\end{array}\right.
$$

Taking a classical linear pressure law, the volumetric potential can then be written:

$$
\rho_{0} \psi_{v o l}=\frac{k}{2}(J-1)^{2} \quad \Rightarrow \quad p=k(J-1)
$$

where $k$ is the compressibility parameter. Therefore, equations (19) become

$$
\left\{\begin{array}{l}
\int_{\Omega_{0}}\left(\boldsymbol{\Pi}_{i s o}(\mathbf{u})+p J \mathbf{F}^{-T}\right): \nabla \delta u d \Omega-\int_{\Omega_{0}} \delta u \cdot \mathbf{f}_{\mathbf{v o l}} d \Omega-\int_{\delta \Omega} \delta u \cdot \mathbf{f}_{\text {surf }} d S \\
\int_{\Omega_{0}}\left(J(\mathbf{u})-1-\frac{p}{k}\right) \delta p d \Omega
\end{array}\right.
$$

It can be seen from the previous equation that $1 / k$ plays the role of a perturbation parameter enforcing incompressibility. The isochoric stress part of the first Piola-Kirchoff stress tensor is computed by simply pulled back the Cauchy stress, so that:

$$
\boldsymbol{\Pi}_{i s o}=J \boldsymbol{\sigma}_{i s o} \cdot \mathbf{F}^{-T}=J\left(\boldsymbol{\sigma}_{e q}+\boldsymbol{\sigma}_{n e q}\right) \cdot \mathbf{F}^{-T}=\boldsymbol{\Pi}_{e q}+\boldsymbol{\Pi}_{n e q}
$$

The tangent moduli calculation and the finite-element implementation are classical and are not described here (see $[16,17]$ and the references therein). The numerical integration of the evolution equation is discussed in $[18,19]$.

\section{Small amplitude loads with large static pre-loads}

The starting point is a simple decomposition of the deformation gradient $\mathbf{F}$ into a static part $\mathbf{F}_{o}$ (which does not depend on time) and a dynamic part $\mathbf{F}_{d}$ (which depends on time):

$$
\mathbf{F}(t)=\mathbf{F}_{d}(t) \cdot \mathbf{F}_{o}
$$




\subsection{Basic assumptions}

The dynamic state is then split into a rotational part and a pure deformation part (see figure 3):

$$
\mathbf{F}=\mathbf{V}_{d} \cdot \mathbf{R}_{d} \cdot \mathbf{F}_{o}=\mathbf{V}_{d} \cdot \tilde{\mathbf{F}}_{o}
$$

The mapping $\tilde{\mathbf{F}}_{o}$ can be said to correspond to a dynamic rotation with preloading. The total change of volume results both from the preload $\mathbf{F}_{o}$ and the dynamic part, $J=J_{o} J_{d}$. The total deformation gradient can be written as follows:

$$
\mathbf{F}=\left(J_{d}^{\frac{1}{3}} \overline{\mathbf{V}}_{d}\right) \cdot\left(J_{o}^{\frac{1}{3}} \tilde{\mathbf{F}}_{o}\right)
$$

The left isochoric Cauchy Green tensor is defined by:

$$
\overline{\mathbf{B}}=\overline{\mathbf{V}}_{d} \cdot \tilde{\mathbf{F}}_{o} \cdot \tilde{\overline{\mathbf{F}}}_{o}^{\mathrm{T}} \cdot \overline{\mathbf{V}}_{d}=\overline{\mathbf{V}}_{d} \cdot \tilde{\overline{\mathbf{B}}}_{o} \cdot \overline{\mathbf{V}}_{d}
$$

The isochoric dynamic pure deformation $\overline{\mathbf{V}}_{d}$ can be split into an elastic part and a viscous part:

$$
\overline{\mathbf{V}}_{d}=\overline{\mathbf{V}}_{e} \cdot \overline{\mathbf{V}}_{v}
$$

with the previous assumption, $\overline{\mathbf{B}}_{\mathbf{e}}$ can be obtain by setting $\overline{\mathbf{V}}_{v}=\mathbf{1}$ and $\overline{\mathbf{F}}_{o}=\mathbf{1}$ in Eq. (26):

$$
\overline{\mathbf{B}}_{\mathbf{e}}=\overline{\mathbf{V}}_{e}^{2}
$$

\subsection{Strain linearization}

The splitting of the dynamic deformation into pure deformation and rotation makes it possible to study the case:

$$
\mathbf{V}_{d}=\mathbf{1}+\boldsymbol{\varepsilon}
$$

where $\boldsymbol{\varepsilon}$ is a small strain deformation tensor. The Taylor expansion of the volume variation is then:

$$
J=J_{o} J_{d}=J_{o} \operatorname{det}(\mathbf{1}+\boldsymbol{\varepsilon})=J_{o}(1+\operatorname{tr} \boldsymbol{\varepsilon})+0\left(\|\boldsymbol{\varepsilon}\|^{2}\right)
$$

The incompressible pure dynamic deformation is:

$$
\begin{aligned}
\overline{\mathbf{V}}_{d} & =J_{d}^{\frac{-1}{3}} \mathbf{V}_{d}=\left(1-\frac{1}{3} \operatorname{tr} \boldsymbol{\varepsilon}+0\left(\|\boldsymbol{\varepsilon}\|^{2}\right)\right)(\mathbf{1}+\boldsymbol{\varepsilon})=\mathbf{1}+\boldsymbol{\varepsilon}-\left(\frac{1}{3} \operatorname{tr} \boldsymbol{\varepsilon}\right) \mathbf{1}+\mathbf{0}\left(\|\boldsymbol{\varepsilon}\|^{2}\right) \\
& =\mathbf{1}+\boldsymbol{\varepsilon}^{\mathrm{D}}+\mathbf{0}\left(\|\boldsymbol{\varepsilon}\|^{2}\right)
\end{aligned}
$$

The Taylor expansion of the incompressible Cauchy-Green tensor can be written:

$$
\begin{aligned}
\overline{\mathbf{B}} & =\left(\mathbf{1}+\boldsymbol{\varepsilon}^{\mathrm{D}}+\mathbf{0}\left(\|\boldsymbol{\varepsilon}\|^{2}\right)\right) \cdot \tilde{\overline{\mathbf{B}}}_{o} \cdot\left(\mathbf{1}+\boldsymbol{\varepsilon}^{\mathrm{D}}+\mathbf{0}\left(\|\boldsymbol{\varepsilon}\|^{2}\right)\right) \\
& =\tilde{\overline{\mathbf{B}}}_{o}+\boldsymbol{\varepsilon}^{\mathrm{D}} \cdot \tilde{\overline{\mathbf{B}}}_{o}+\tilde{\overline{\mathbf{B}}}_{o} \cdot \boldsymbol{\varepsilon}^{\mathrm{D}}+\mathbf{0}\left(\|\boldsymbol{\varepsilon}\|^{2}\right)
\end{aligned}
$$

and its square is:

$$
\overline{\mathbf{B}}^{2}=\tilde{\overline{\mathbf{B}}}_{o}^{2}+\boldsymbol{\varepsilon}^{\mathrm{D}} \cdot \tilde{\overline{\mathbf{B}}}_{o}^{2}+\tilde{\overline{\mathbf{B}}}_{o}^{2} \cdot \boldsymbol{\varepsilon}^{\mathrm{D}}+2 \tilde{\overline{\mathbf{B}}}_{o} \cdot \boldsymbol{\varepsilon}^{\mathrm{D}} \cdot \tilde{\overline{\mathbf{B}}}_{o}+\mathbf{0}\left(\|\boldsymbol{\varepsilon}\|^{2}\right)
$$

Assuming that $\overline{\mathbf{V}}_{e}=\mathbf{1}+\boldsymbol{\varepsilon}_{e}^{\mathrm{D}}$ and $\overline{\mathbf{V}}_{v}=\mathbf{1}+\boldsymbol{\varepsilon}_{v}^{\mathrm{D}}$, we obtain the classical definition: $\boldsymbol{\varepsilon}=\boldsymbol{\varepsilon}_{e}+\boldsymbol{\varepsilon}_{v}$ and the elastic linearized Cauchy-Green tensor is:

$$
\overline{\mathbf{B}}_{e}=\left(\mathbf{1}+\boldsymbol{\varepsilon}_{e}^{\mathrm{D}}+\mathbf{0}\left(\left\|\boldsymbol{\varepsilon}_{e}\right\|^{2}\right)\right)^{2}=\mathbf{1}+2 \boldsymbol{\varepsilon}_{e}^{\mathrm{D}}+\mathbf{0}\left(\left\|\boldsymbol{\varepsilon}_{e}\right\|^{2}\right)
$$

and its time variation is:

$$
\dot{\overline{\mathbf{B}}}_{e}=2 \dot{\boldsymbol{\varepsilon}}_{e}^{\mathrm{D}}+\mathbf{0}\left(\left\|\dot{\boldsymbol{\varepsilon}}_{e}\right\|^{2}\right)
$$


The Taylor expansion of the velocity gradient is defined by:

$$
\begin{aligned}
\mathbf{L} & =\frac{\cdot}{(\mathbf{1}+\boldsymbol{\varepsilon}) \cdot \tilde{\mathbf{F}}_{o}} \cdot \tilde{\mathbf{F}}_{o}^{-1} \cdot(\mathbf{1}+\boldsymbol{\varepsilon})^{-1} \\
& =\dot{\boldsymbol{\varepsilon}} \cdot\left(\mathbf{1}-\boldsymbol{\varepsilon}+\mathbf{0}(\|\boldsymbol{\varepsilon}\|)^{2}\right)+(\mathbf{1}+\boldsymbol{\varepsilon}) \cdot \dot{\tilde{\mathbf{F}}}_{o} \cdot \tilde{\mathbf{F}}_{o}^{-1} \cdot\left(\mathbf{1}-\boldsymbol{\varepsilon}+\mathbf{0}\left(\|\boldsymbol{\varepsilon}\|^{2}\right)\right) \\
& =\dot{\boldsymbol{\varepsilon}}+\tilde{\mathbf{L}}_{o}+\boldsymbol{\varepsilon} \cdot \tilde{\mathbf{L}}_{o}-\tilde{\mathbf{L}}_{o} \cdot \boldsymbol{\varepsilon}+\mathbf{0}\left(\|\boldsymbol{\varepsilon}\|^{2}\right)
\end{aligned}
$$

Where $\dot{\tilde{\mathbf{F}}}_{o}=\dot{\mathbf{R}}_{d} \cdot \mathbf{F}_{o}$. In the case of a null rotational dynamic deformation or a constant one, the linearized velocity gradient is: $\mathbf{L}=\dot{\boldsymbol{\varepsilon}}$ (as $\mathbf{F}_{o}$ does not depend on time). In what follows, we will deal only with isotropic behaviors. Therefore, the free specific energy is only a function of the first two invariants of the incompressible Cauchy-Green tensor. By definition, we have:

$$
\begin{aligned}
I_{1}(\overline{\mathbf{B}})= & \mathbf{1}: \overline{\mathbf{B}}=I_{1}\left(\tilde{\overline{\mathbf{B}}}_{o}\right)+2\left(\boldsymbol{\varepsilon}^{\mathrm{D}}: \tilde{\overline{\mathbf{B}}}_{o}\right)+\mathbf{0}\left(\|\boldsymbol{\varepsilon}\|^{2}\right) \\
I_{2}(\overline{\mathbf{B}})= & \frac{1}{2}\left(I_{1}(\overline{\mathbf{B}})^{2}-\mathbf{1}: \overline{\mathbf{B}}^{2}\right) \\
= & \frac{1}{2}\left(I_{1}\left(\tilde{\overline{\mathbf{B}}}_{o}\right)+2\left(\boldsymbol{\varepsilon}^{\mathrm{D}}: \tilde{\overline{\mathbf{B}}}_{o}\right)+\mathbf{0}\left(\|\boldsymbol{\varepsilon}\|^{2}\right)\right. \\
& \left.\quad\left(\tilde{\overline{\mathbf{B}}}_{o}+\boldsymbol{\varepsilon}^{\mathrm{D}} \cdot \tilde{\overline{\mathbf{B}}}_{o}+\tilde{\overline{\mathbf{B}}}_{o} \cdot \boldsymbol{\varepsilon}^{\mathrm{D}}\right):\left(\tilde{\overline{\mathbf{B}}}_{o}+\boldsymbol{\varepsilon}^{\mathrm{D}} \cdot \tilde{\overline{\mathbf{B}}}_{o}+\tilde{\overline{\mathbf{B}}}_{o} \cdot \boldsymbol{\varepsilon}^{\mathrm{D}}\right)\right) \\
= & I_{2}\left(\tilde{\mathbf{B}}_{o}\right)+\left(\boldsymbol{\varepsilon}^{\mathrm{D}}: \tilde{\mathbf{B}}_{o}\right)-2\left(\boldsymbol{\varepsilon}^{\mathrm{D}}: \tilde{\mathbf{B}}_{o}\right)+\mathbf{0}\left(\|\boldsymbol{\varepsilon}\|^{2}\right)
\end{aligned}
$$

In addition, due to orthogonality properties, we have:

$$
I_{1}\left(\tilde{\overline{\mathbf{B}}}_{o}\right)=I_{1}\left(\overline{\mathbf{B}}_{o}\right) \quad \text { and } \quad I_{2}\left(\tilde{\overline{\mathbf{B}}}_{o}\right)=I_{2}\left(\overline{\mathbf{B}}_{o}\right)
$$

\subsection{Stress linearization}

The stress in the Zener model is defined in Eq. (10) and as mentioned above, the model is assumed to show an isotropic behavior and the deviatoric Piola-Kirchoff equilibrium stress becomes:

$$
\boldsymbol{\Pi}_{e q}=J \boldsymbol{\sigma}_{e q} \mathbf{F}^{-T}=2 \rho_{0}\left[\overline{\mathbf{B}}\left(\frac{\partial \psi_{e q}}{\partial I_{1}}-I_{1} \frac{\partial \psi_{e q}}{\partial I_{2}}\right)-\overline{\mathbf{B}}^{2} \frac{\partial \psi_{e q}}{\partial I_{2}}\right]^{\mathrm{D}} \cdot \mathbf{F}^{-T}
$$

$\psi_{e q, 1}^{o}$ and $\psi_{e q, 1}^{\boldsymbol{\varepsilon}}$ are taken to denote the partial derivative of $\psi_{e q}$ with respect to $I_{1}$ that depend on $\boldsymbol{\varepsilon}^{0}$ and $\boldsymbol{\varepsilon}^{1}$.

$$
\begin{aligned}
\boldsymbol{\Pi}_{e q}= & 2 \rho_{0}\left[\left(\tilde{\overline{\mathbf{B}}}_{o}+\boldsymbol{\varepsilon}^{\mathrm{D}} \cdot \tilde{\overline{\mathbf{B}}}_{o}+\tilde{\overline{\mathbf{B}}}_{o} \cdot \boldsymbol{\varepsilon}^{\mathrm{D}}\right)\left(\psi_{e q, 1}^{o}+\psi_{e q, 1}^{\boldsymbol{\varepsilon}}-\left(I_{1}\left(\overline{\mathbf{B}}_{o}\right)+2\left(\boldsymbol{\varepsilon}^{\mathrm{D}}: \tilde{\overline{\mathbf{B}}}_{o}\right)\right)\left(\psi_{e q, 2}^{o}+\psi_{e q, 2}^{\boldsymbol{\varepsilon}}\right)\right)\right. \\
& \left.-\left(\tilde{\overline{\mathbf{B}}}_{o}+\boldsymbol{\varepsilon}^{\mathrm{D}} \cdot \tilde{\overline{\mathbf{B}}}_{o}^{2}+\tilde{\overline{\mathbf{B}}}_{o}^{2} \cdot \boldsymbol{\varepsilon}^{\mathrm{D}}+2 \tilde{\overline{\mathbf{B}}}_{o} \cdot \boldsymbol{\varepsilon}^{\mathrm{D}} \cdot \tilde{\overline{\mathbf{B}}}_{o}\right)\left(\psi_{e q, 2}^{o}+\psi_{e q, 2}^{\boldsymbol{\varepsilon}}\right)\right]^{\mathrm{D}} \cdot(\mathbf{1}-\boldsymbol{\varepsilon}) \cdot \tilde{\mathbf{F}}_{o}^{-T} \\
= & \tilde{\boldsymbol{\tau}}_{o} \cdot \mathbf{\mathbf { F }}_{0}^{-T}-\tilde{\boldsymbol{\tau}}_{o} \cdot \boldsymbol{\varepsilon} \cdot \tilde{\mathbf{F}}_{o}^{-T}+2 \rho_{0}\left[\left(\boldsymbol{\varepsilon}^{\mathrm{D}} \cdot \tilde{\overline{\mathbf{B}}}_{o}+\tilde{\overline{\mathbf{B}}}_{o} \cdot \boldsymbol{\varepsilon}^{\mathrm{D}}\right)\left(\psi_{e q, 1}^{o}-I_{1}\left(\overline{\mathbf{B}}_{o}\right) \psi_{e q, 2}^{o}\right)\right. \\
& \left.-2\left(\boldsymbol{\varepsilon}^{\mathrm{D}}: \tilde{\overline{\mathbf{B}}}_{o}\right) \psi_{e q, 2}^{o} \tilde{\overline{\mathbf{B}}}_{o}-\left(\boldsymbol{\varepsilon}^{\mathrm{D}} \cdot \tilde{\overline{\mathbf{B}}}_{o}^{2}+\tilde{\overline{\mathbf{B}}}_{o}^{2} \cdot \boldsymbol{\varepsilon}^{\mathrm{D}}+2 \tilde{\overline{\mathbf{B}}}_{o} \cdot \boldsymbol{\varepsilon}^{\mathrm{D}} \cdot \tilde{\overline{\mathbf{B}}}_{o}\right) \psi_{e q, 2}^{o}\right]^{\mathrm{D}} \cdot \tilde{\mathbf{F}}_{o}^{-T} \\
& +2 \rho_{0}\left[\tilde{\overline{\mathbf{B}}}_{o}\left(\psi_{e q, 1}^{\boldsymbol{\varepsilon}}-I_{1}\left(\overline{\mathbf{B}}_{o}\right) \psi_{e q, 2}^{\boldsymbol{\varepsilon}}\right)-\tilde{\overline{\mathbf{B}}}_{o}^{2} \psi_{e q, 2}^{\boldsymbol{\varepsilon}}\right]^{\mathrm{D}} \cdot \tilde{\mathbf{F}}_{o}^{-T}+\mathbf{0}\left(\|\boldsymbol{\varepsilon}\|^{2}\right)
\end{aligned}
$$

where $\tilde{\boldsymbol{\tau}}_{o}$ is defined by:

$$
\begin{aligned}
\tilde{\boldsymbol{\tau}}_{o} & =2 \rho_{0}\left[\tilde{\overline{\mathbf{B}}}_{o}\left(\psi_{e q, 1}^{o}-I_{1}\left(\overline{\mathbf{B}}_{o}\right) \psi_{e q, 2}^{o}\right)-\tilde{\overline{\mathbf{B}}}_{o} \psi_{e q, 2}^{o}\right]^{\mathrm{D}} \\
& =2 \rho_{0} \mathbf{R}_{d} \cdot\left[\overline{\mathbf{B}}_{o}\left(\psi_{e q, 1}^{o}-I_{1}\left(\overline{\mathbf{B}}_{o}\right) \psi_{e q, 2}^{o}\right)-\overline{\mathbf{B}}_{o}^{2} \psi_{e q, 2}^{o}\right]^{\mathrm{D}} \cdot \mathbf{R}_{d}^{-T} \\
& =\mathbf{R}_{d} \cdot \tau_{o} \cdot \mathbf{R}_{d}^{-T}
\end{aligned}
$$


The determination of the Taylor expansion of the non-equilibrium stress follows the same lines:

$$
\begin{aligned}
\boldsymbol{\Pi}_{n e q} & =2 \rho_{0}\left[\overline{\mathbf{B}}_{\mathbf{e}}\left(\frac{\partial \psi_{n e q}}{\partial I_{1}^{e}}-I_{1}\left(\overline{\mathbf{B}}_{\mathbf{e}}\right) \frac{\partial \psi_{n e q}}{\partial I_{2}^{e}}\right)-\overline{\mathbf{B}}_{\mathbf{e}}{ }^{2} \frac{\partial \psi_{n e q}}{\partial I_{2}^{e}}\right]^{\mathrm{D}} \cdot \mathbf{F}^{-T} \\
& =2 \rho_{0}\left[\left(\mathbf{1}+2 \boldsymbol{\varepsilon}_{e}^{\mathrm{D}}\right)\left(\psi_{e q, 1^{e}}^{o}+\psi_{e q, 1^{e}}^{\boldsymbol{\varepsilon}}-3 \psi_{e q, 2^{e}}^{o}-3 \psi_{e q, 2^{e}}^{\varepsilon}\right)-\left(\mathbf{1}+4 \boldsymbol{\varepsilon}_{e}^{\mathrm{D}}\right)\left(\psi_{e q, 2^{e}}^{o}+\psi_{e q, 2^{e}}^{\boldsymbol{\varepsilon}}\right)\right]^{\mathrm{D}} \cdot(\mathbf{1}-\boldsymbol{\varepsilon}) \cdot \tilde{\mathbf{F}}_{o}^{-T} \\
& =4 \rho_{0}\left[\boldsymbol{\varepsilon}_{e}^{\mathrm{D}}\left(\psi_{e q, 1^{e}}^{o}-5 \psi_{e q, 2^{e}}^{o}\right)\right] \cdot \tilde{\mathbf{F}}_{o}^{-T}+\mathbf{0}\left(\left\|\boldsymbol{\varepsilon}_{e}\right\|^{2}\right)
\end{aligned}
$$

Therefore, the total stress is not defined as a sum of the static ones and a dynamic one. The evolution equation (14), can also be linearized using the expression developed in the previous section:

$$
\begin{aligned}
2 \dot{\boldsymbol{\varepsilon}}_{e}^{\mathrm{D}} & =\left(\dot{\boldsymbol{\varepsilon}}+\tilde{L}_{o}+\boldsymbol{\varepsilon} \cdot \tilde{L}_{o}-\tilde{L}_{o} \cdot \boldsymbol{\varepsilon}\right) \cdot\left(\mathbf{1}+2 \boldsymbol{\varepsilon}_{e}^{\mathrm{D}}\right)+\left(\mathbf{1}+2 \boldsymbol{\varepsilon}_{e}^{\mathrm{D}}\right) \cdot\left(\dot{\boldsymbol{\varepsilon}}+\tilde{L}_{o}+\boldsymbol{\varepsilon} \cdot \tilde{L}_{o}-\tilde{L}_{o} \cdot \boldsymbol{\varepsilon}\right)^{\mathrm{T}} \\
& -\frac{2}{3}\left(\left(\dot{\boldsymbol{\varepsilon}}+\tilde{L}_{o}+\boldsymbol{\varepsilon} \cdot \tilde{L}_{o}-\tilde{L}_{o} \cdot \boldsymbol{\varepsilon}\right): \mathbf{1}\right)\left(\mathbf{1}+2 \boldsymbol{\varepsilon}_{e}^{\mathrm{D}}\right)-\frac{2}{\eta} \boldsymbol{\sigma}_{n e q} \cdot\left(\mathbf{1}+2 \boldsymbol{\varepsilon}_{e}^{\mathrm{D}}\right)
\end{aligned}
$$

and therefore:

$$
\dot{\boldsymbol{\varepsilon}}_{e}^{\mathrm{D}}=\dot{\boldsymbol{\varepsilon}}^{\mathrm{D}}+\tilde{\mathbf{D}}_{o}^{\mathrm{D}}+\left(\boldsymbol{\varepsilon} \cdot \tilde{\mathbf{W}}_{o}-\tilde{\mathbf{W}}_{o} \cdot \boldsymbol{\varepsilon}\right)+\left(\boldsymbol{\varepsilon}_{e}^{\mathrm{D}} \cdot \tilde{\mathbf{L}}_{o}+\tilde{\mathbf{L}}_{o} \cdot \boldsymbol{\varepsilon}_{e}^{\mathrm{D}}\right)-\frac{2}{3}\left(\tilde{L}_{o}: \mathbf{1}\right) \boldsymbol{\varepsilon}_{e}^{\mathrm{D}}-\frac{1}{\eta} \boldsymbol{\sigma}_{n e q}+\mathbf{0}\left(\left\|\boldsymbol{\varepsilon}_{e}\right\|^{2}\right)+\mathbf{0}\left(\|\boldsymbol{\varepsilon}\|^{2}\right)
$$

where $\boldsymbol{\sigma}_{n e q}$ is defined from:

$$
\boldsymbol{\sigma}_{n e q}=4 \rho_{0} J_{o}^{-1}\left[\boldsymbol{\varepsilon}_{e}^{\mathrm{D}}\left(\psi_{e q, 1^{e}}^{o}-5 \psi_{e q, 2^{e}}^{o}\right)\right]+\mathbf{0}\left(\left\|\boldsymbol{\varepsilon}_{e}\right\|^{2}\right)
$$

and $\tilde{\mathbf{D}}_{o}, \tilde{\mathbf{W}}_{o}$ are the symmetric and antisymmetric parts ${ }^{2}$ of $\tilde{\mathbf{L}}_{o}$.

\subsection{Flow rule integration}

Let us take the time interval $\left[t_{n}, t_{n+1}\right]$. Given ${ }^{3}$ the quantities known at $t_{n}$ and the predicted quantity $\mathbf{R}_{d}^{n+1}, \boldsymbol{\varepsilon}^{n+1}$, a simple backward Euler scheme is used to integrate Eq. (45). The time derivatives are evaluated as follows:

$$
\begin{aligned}
\tilde{\mathbf{L}}_{o} & =\dot{\mathbf{R}}_{d} \cdot \mathbf{R}_{d}^{\mathrm{T}}=\frac{\mathbf{R}_{d}^{n+1}-\mathbf{R}_{d}^{n}}{\Delta t} \cdot \mathbf{R}_{d}^{\mathrm{T}} \\
\dot{\boldsymbol{\varepsilon}} & =\frac{\boldsymbol{\varepsilon}^{n+1}-\boldsymbol{\varepsilon}^{n}}{\Delta t} \\
\dot{\boldsymbol{\varepsilon}}_{e} & =\frac{\boldsymbol{\varepsilon}_{e}^{n+1}-\boldsymbol{\varepsilon}_{e}^{n}}{\Delta t}
\end{aligned}
$$

Using the previous equation (45) and evaluating all the quantities at time $t_{n+1}$, the following value of the deviatoric part of $\boldsymbol{\varepsilon}_{e}^{n+1}$ is obtained:

$$
\boldsymbol{\varepsilon}_{e}^{\mathrm{D}^{n+1}}=\mathbb{A}^{-1}:\left(\frac{1}{\Delta t} \boldsymbol{\varepsilon}_{e}^{\mathrm{D}^{n}}+\frac{\boldsymbol{\varepsilon}^{\mathrm{D}^{n+1}}-\boldsymbol{\varepsilon}^{\mathrm{D}^{n}}}{\Delta t}+\boldsymbol{\varepsilon}^{n+1} \cdot \tilde{\mathbf{W}}_{o}^{n+1}-\tilde{\mathbf{W}}_{o}^{n+1} \cdot \boldsymbol{\varepsilon}^{n+1}\right)
$$

where $\mathbb{A}$ is a fourth order tensor defined by ${ }^{4}$ :

$$
\mathbb{A}=\left(\frac{1}{\Delta t}+\frac{4 \rho_{0}}{J_{o} \eta}\left(\psi_{e q, 1^{e}}^{o}-5 \psi_{e q, 2^{e}}^{o}\right)+\frac{2}{3}\left(\tilde{L}_{o}^{n+1}: \mathbf{1}\right)\right) \mathbb{I}-\mathbf{1} \oplus \tilde{\mathbf{L}}_{o}^{n+1}-\tilde{\mathbf{L}}_{o}^{n+1} \oplus \mathbf{1}
$$

where $\mathbb{I}$ stands for the fourth order identity tensor.

\footnotetext{
${ }^{2} \tilde{\mathbf{D}}_{o}=\frac{1}{2}\left(\tilde{L}_{o}+\tilde{L}_{o}^{\mathrm{T}}\right) \quad \tilde{\mathbf{W}}_{o}=\frac{1}{2}\left(\tilde{L}_{o}-\tilde{L}_{o}^{\mathrm{T}}\right)$

${ }^{3}$ The superscripts ${ }^{n+1}$ and ${ }^{n}$ stands for the evaluation at times $t_{n+1}$ and $t_{n}$, respectively.

${ }^{4}$ The tensorial symbol $\oplus$ stands for: $\mathbb{A}_{i j k l}=B_{i k} C_{j l}=\mathbf{B} \oplus \mathbf{C}$.
} 


\subsection{Numerical implementation}

This linearized model has been implemented in the finite element software ZeBuLoN developed by Ecole des Mines de Paris, ONERA and Northwest Numerics. This object-oriented software is written in C++ and is suitable for developing variational formulation, user defined elements and constitutive laws (see [20]). The computation of a linearized case with a large preload is decomposed in two or more steps. The first one deals with the large static preload and the second one is a dynamic (quasi-static) step. The object-oriented aspect makes it posible to derive a linearized Zener model from an existing class of hyperelastic laws. Therefore, the computation of the tangent modulus part resulting from the equilibrium stress in the Zener model is quite straightforward, as explained below.

We note $\mathbb{C}$ the consistent operator:

$$
\mathbb{C}=\underbrace{\frac{\partial \boldsymbol{\Pi}_{e q}}{\partial \mathbf{F}}}_{\mathbb{C}_{e q}}+\underbrace{\frac{\partial \boldsymbol{\Pi}_{n e q}}{\partial \mathbf{F}}}_{\mathbb{C}_{n e q}}+p \underbrace{\frac{\partial J \mathbf{F}^{-T}}{\partial \mathbf{F}}}_{\mathbb{C}_{v o l}}
$$

In the linearization step, the consistent operators $\mathbb{C}_{e q}$ and $\mathbb{C}_{v o l}$ are deduced from their general expression at finite strain by keeping only $0^{\text {th }}$ order terms in $\boldsymbol{\varepsilon}$. Given $\mathbf{F}=(1+\boldsymbol{\varepsilon}) \cdot \tilde{\mathbf{F}}_{o}$, the gradient $\mathbf{F}$ is replaced by $\tilde{\mathbf{F}}_{o}$ in the expressions for the tangent moduli (see $[16,17]$ for detailed expressions for $\mathbb{C}_{e q}$ and $\mathbb{C}_{v o l}$ ).

We proposed an approximate $\mathbb{C}_{n e q}$ focusing on the small strain formulation:

$$
\mathbb{C}_{n e q} \approx \underbrace{\frac{\partial \boldsymbol{\Pi}_{n e q}}{\partial \boldsymbol{\varepsilon}_{e}^{\mathrm{D}}}}_{\mathbb{C}_{\boldsymbol{\varepsilon}_{e}}}: \underbrace{\frac{\partial \boldsymbol{\varepsilon}_{e}^{\mathrm{D}}}{\partial \boldsymbol{\varepsilon}^{\mathrm{D}}}}_{\mathbb{H}}: \underbrace{\frac{\partial \boldsymbol{\varepsilon}^{\mathrm{D}}}{\partial \mathbf{F}}}_{\mathbb{K}}
$$

A direct computation gives the first term:

$$
\mathbb{C}_{\boldsymbol{\varepsilon}_{e}}=4 \rho_{0}\left(\psi_{e q, 1^{e}}^{o}-5 \psi_{e q, 2^{e}}^{o}\right) \mathbf{1} \oplus \tilde{\mathbf{F}}_{o}^{-1}
$$

The second term is obtained by computing the first variation of the flow rule with respect to $\boldsymbol{\varepsilon}^{\mathrm{D}}$. Starting with Eq. (50) and neglecting the terms $\partial \boldsymbol{\varepsilon}_{e}^{\mathrm{D}^{n}} / \partial \boldsymbol{\varepsilon}^{\mathrm{D}}$ or $\partial \boldsymbol{\varepsilon}^{\mathrm{D}^{n}} / \partial \boldsymbol{\varepsilon}^{\mathrm{D}}$, we obtain the following expression:

$$
\mathbb{H}=\mathbb{A}^{-1}:\left(\frac{1}{\Delta t} \mathbb{I}-\mathbf{1} \oplus \tilde{\mathbf{W}}_{o}^{n+1}-\tilde{\mathbf{W}}_{o}^{n+1} \oplus \mathbf{1}\right)
$$

For the last term, we have:

$$
\boldsymbol{\varepsilon}=\mathbf{F} \cdot \tilde{\mathbf{F}}_{o}^{-1}-\mathbf{1} \Rightarrow \boldsymbol{\varepsilon}^{\mathrm{D}}=\left(\mathbf{F} \cdot \tilde{\mathbf{F}}_{o}^{-1}\right)^{\mathrm{D}}
$$

therefore, $\mathbb{K}$ is given by:

$$
\mathbb{K}=\mathbf{1} \oplus \tilde{\mathbf{F}}_{o}^{-T}-\frac{1}{3} \mathbf{1} \otimes \tilde{\mathbf{F}}_{o}^{-T}
$$

where $\otimes$ stands for the standard tensorial product.

We use quadratic elements Q9P3 for plane strain and C27P4 for 3D problems. These elements are based on quadratic shape functions for the kinematic. A linear interpolation is used for the pressure field (see fig. 4). These choices combined with a full Gauss integration ensure stable elements in the sense of the LBB condition (see [21, 22] and the references theirein). Furthermore as the pressure field does not have to be continuous between elements a static condensation of the pressure degrees of freedom is performed inside the elements.

\section{Numerical applications}

\subsection{Rectangular rubber block submitted to traction or torsion}

In this section, we propose to compare the results obtained with the present model with those obtained using a finite strain model or a linarization strategy such as that developed by Höfer and Lion [8]. In the latter paper, the authors adopted the following decomposition:

$$
\mathbf{F}=(\mathbf{1}+\mathbf{h}) \cdot \mathbf{F}_{o} \quad \text { and } \quad \boldsymbol{\varepsilon}=1 / 2\left(\mathbf{h}+\mathbf{h}^{\mathrm{T}}\right)
$$




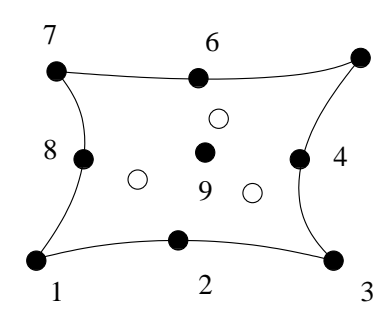

(a) Q9P3

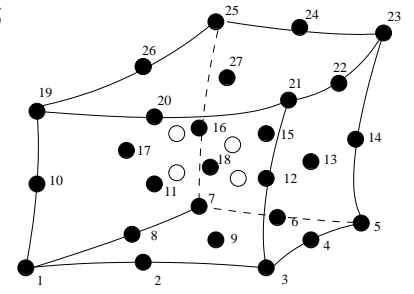

(b) $\mathrm{C} 27 \mathrm{P} 4$

Figure 4: Two examples of finite element used in the F.E. code ZeBuLoN. Kinematic nodes are designed by filled dots, pressure nodes by unfilled ones

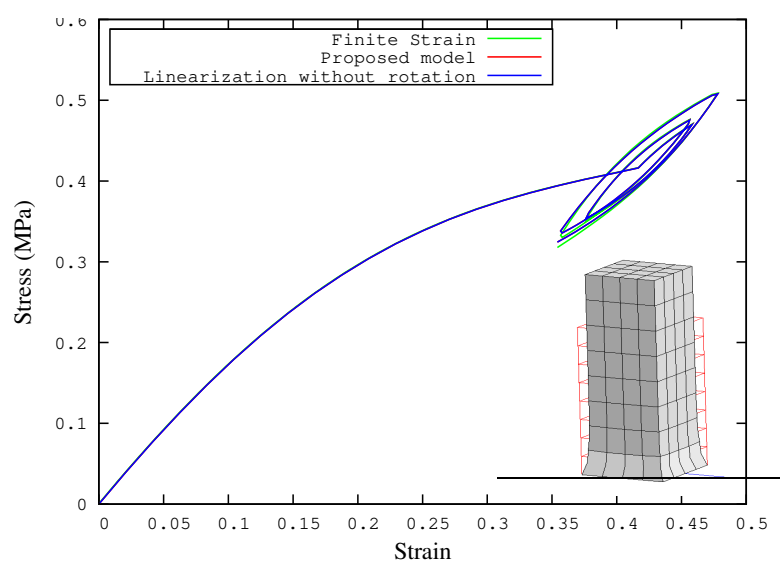

(a) Static and dynamic responses.

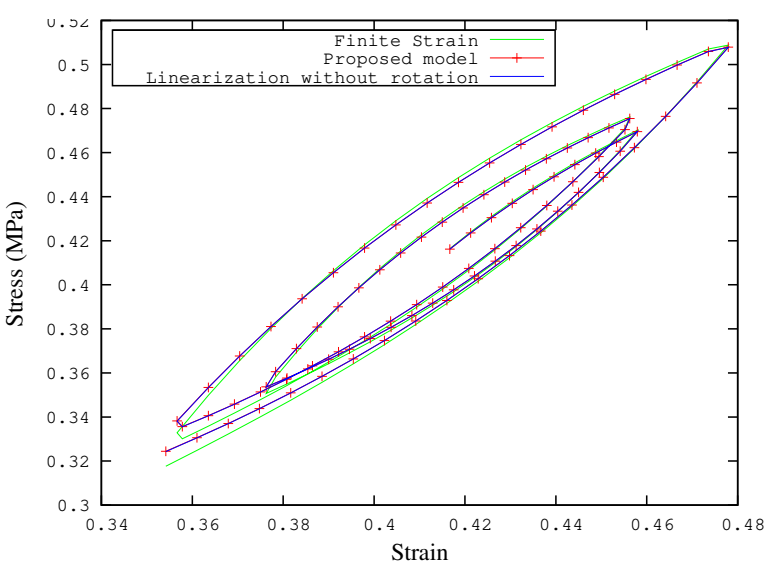

(b) Zoom on the dynamic response.

Figure 5: Extension of a rectangular block of rubber. The finite strain model, the present model and a linearization without rotations are compared.

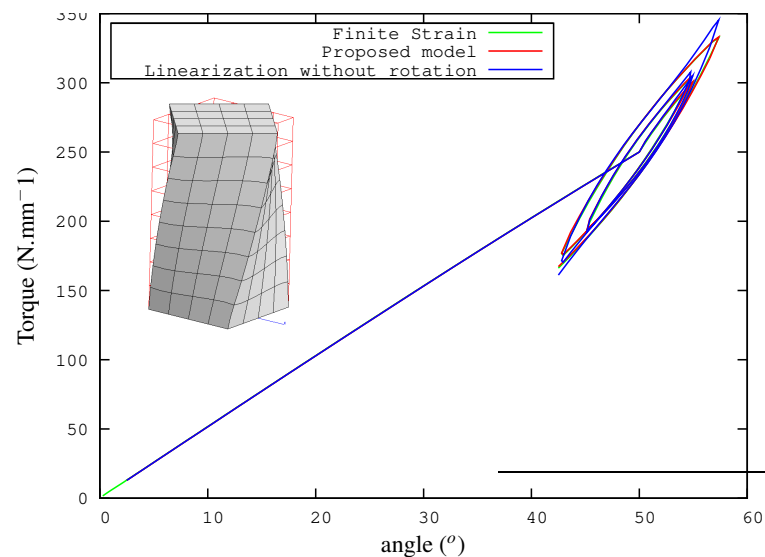

(a) Static and dynamic responses.

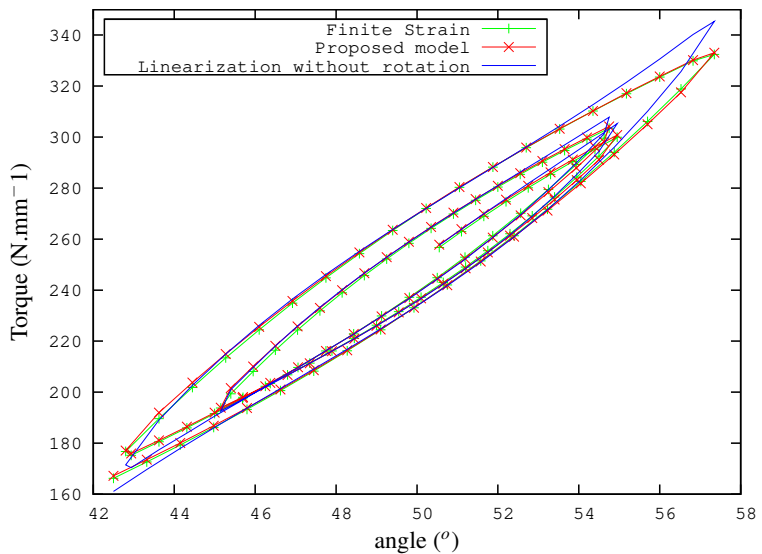

(b) Zoom on the dynamic response.

Figure 6: Torsion of a rectangular block of rubber. The finite strain model, the present model and a linearization without rotations are compared.

All the terms in $\mathbf{h}^{2}$ were assumed to be negligible. In what follows, the results obtained with the decomposition of Eq. (58) will be referred to as "linearization without rotation".

The numerical tests are performed on a rectangular block of rubber, the upper surface of which is subjected to the following loads: 
- case (a): small amplitude dynamic torsion angle combined with a static torsion state,

- case (b): small amplitude dynamic vertical displacement combined with a static one.

In both cases, the lower surface is fixed and the lateral surfaces are force-free. The cross-section of the rectangular block measures $30 \times 30 \mathrm{~mm}$ and the block is $150 \mathrm{~mm}$ long. In the torsion test, the torsion angle is imposed at a reference point controlling the rigid motion of the top surface. The lateral displacements and the other rotational degrees of freedom of this point are fixed, whereas the vertical translation is free. In the traction test, the top surface is subjected to an imposed vertical displacement with free lateral displacements. In both the torsion and traction tests, the dynamic load is applied via a triangular signal with a frequency of $5 \mathrm{~Hz}$. The following hyperelastic potentials are adopted:

$$
\left\{\begin{array}{l}
\psi_{\text {eq }}(\overline{\mathbf{B}})=c_{10}\left(I_{1}(\overline{\mathbf{B}})-3\right)+c_{01}\left(I_{2}(\overline{\mathbf{B}})-3\right)+c_{20}\left(I_{1}(\overline{\mathbf{B}})-3\right)^{2}+c_{30}\left(I_{1}(\overline{\mathbf{B}})-3\right)^{3} \\
\psi_{\text {neq }}\left(\overline{\mathbf{B}}_{\mathbf{e}}\right)=G\left(I_{1}\left(\overline{\mathbf{B}}_{\mathbf{e}}\right)-3\right) \\
\psi_{\text {vol }}(J)=\frac{k}{2}(J-1)^{2}
\end{array}\right.
$$

The material parameters are given in table 1 . The mesh comprises 128 quadratic elements (Q2P1).

\begin{tabular}{llllllll}
\hline & $c_{10}(\mathrm{MPa})$ & $c_{01}(\mathrm{MPa})$ & $c_{20}(\mathrm{MPa})$ & $c_{30}(\mathrm{MPa})$ & $G(\mathrm{MPa})$ & $\eta(\mathrm{MPa} . \mathrm{s})$ & $\mathrm{k}(\mathrm{MPa})$ \\
\hline Zener & 0.090 & -0.014 & -0.042 & 0.019 & 0.110 & 0.030 & 1500 \\
\hline
\end{tabular}

Table 1: Material parameters used in all the models.

Figure 5(a) gives the static and dynamic responses of the traction test with the three models. The two linearized models yield very similar results, which are also similar to those obtained with the finite strain model. As shown in figure 5(b), with a dynamic amplitude of $4 \%$, the dynamic response is the same with all three models. At 6\%, a small difference is observed in the unloading path.

The results obtained in the torsion test show that the two linearized models give a different behavior: as shown in figures 6(a) and 6(b) the "linearized model without rotation" gives a stiffer response than the finite strain model, which is not so in the case of the present model. The present model can therefore be said to be accurate at large rotations.

\subsection{Shear test on a rubber mounting}

This example is inspired from a sandwich rubber mounting used in a space rocket. This mounting is designed to isolate the central part of the structure during launch phase, it is mainly submitted to an instantaneous shear loading

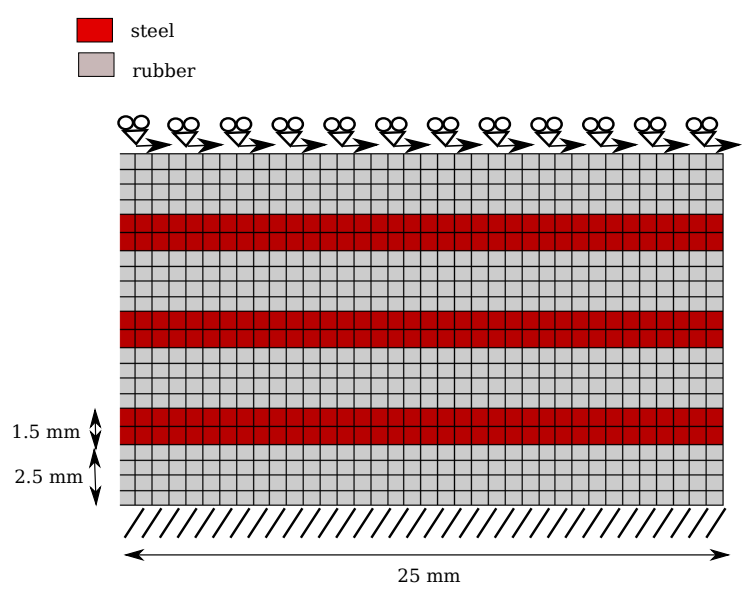

Figure 7: Meshing and boundaries conditions of a sandwich rubber mounting. The finite element mesh comprises Q2P1 elements for rubber and Q2 elements for steel. 


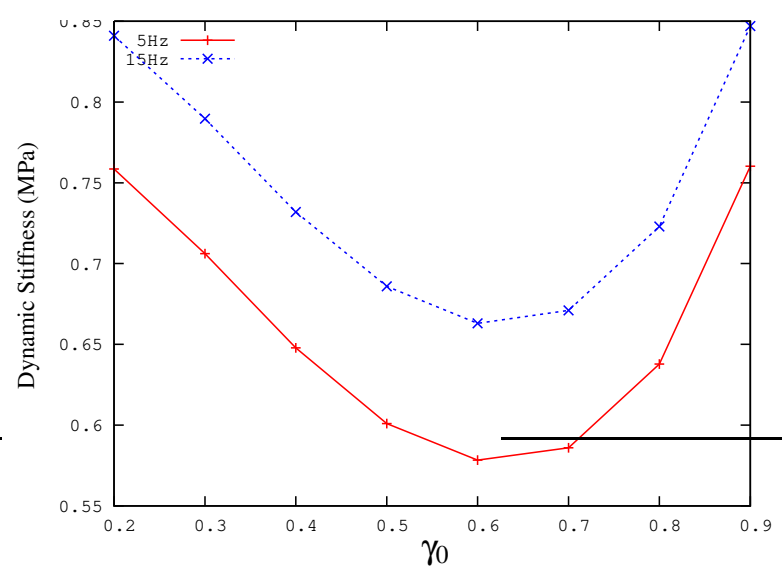

(a) Static and dynamic responses.

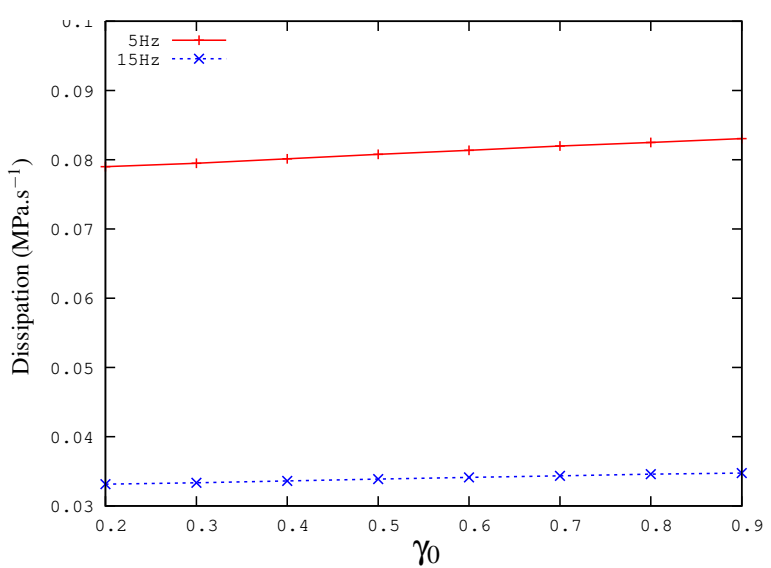

(b) Zoom on the dynamic response.

Figure 8: Shearing test on the rubber mounting using the proposed model. Effects of preloading amplitude and frequency of the small vibrations are shown.

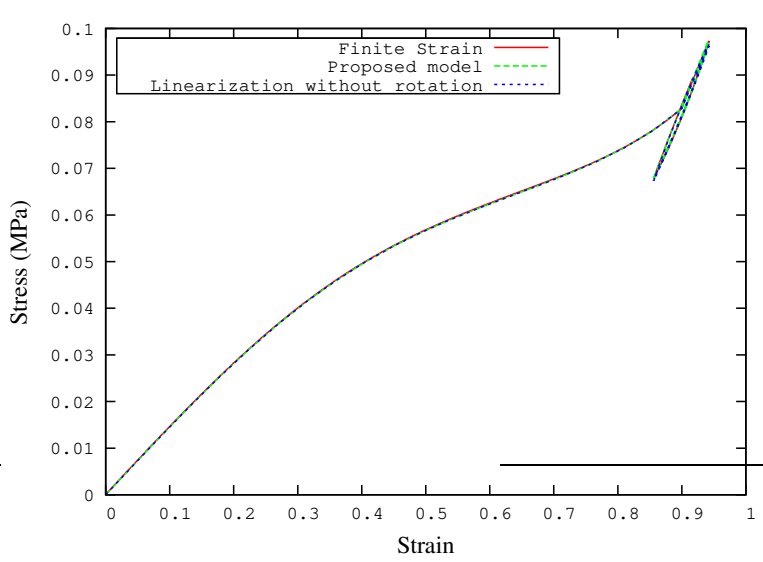

(a) Static and dynamic responses.

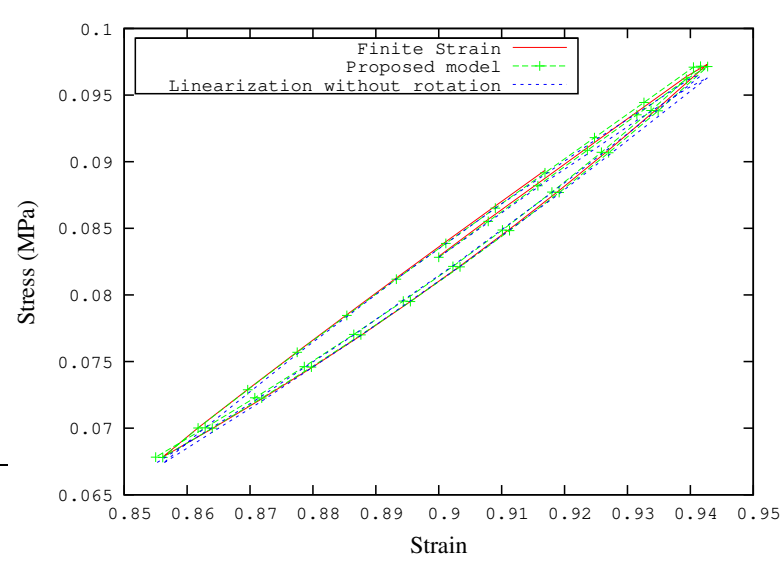

(b) Zoom on dynamic response.

Figure 9: Comparison between shear tests on the rubber mounting based on the finite strain model, the present model and a linearization without rotations $\left(\gamma_{0}=0.9, f=15 \mathrm{~Hz}\right)$.

(considered as static) overloaded by small oscillatory shear loads. The design of this part is critical for both, the health of equipments embed in the central part of the rocket, and, the control of the propulsion during launching. In this application, the non-linear dependency of the storage and the loss modulus upon the static preload is the key point for the engineers. We consider here a sandwich composed by 4 layers of rubber $\left(2.5 \times 25 \mathrm{~mm}^{2}\right)$ separated by 3 layers of steel $\left(1.5 \times 25 \mathrm{~mm}^{2}\right)$. It is assumed to be in a plane strain state. The bottom layer is fixed, whereas the upper one is subjected to a transversal displacement (no vertical displacement is allowed). The geometry and the boundary conditions are shown in figure 7. The material parameters of rubber are given in table 1 . The steel is assumed to be elastic and isotropic: $E=200000 \mathrm{Mpa}$ and $v=0.3$. The shear (transversal) displacement is imposed in two steps. In the first (static) step, the shear rate is very slow. The amplitude $\gamma_{0}$ is defined as the total height of the rubber divided by the transversal displacement applied. In the first step, the assumption: $\gamma_{0} \in[0,1]$ is made. In the second (quasi-static) step, a small amplitude $(\gamma=0.05)$ dynamic transversal displacement is imposed (the dynamic signal is triangular).

The dynamic shear stiffness and the global dissipation occurring during a cycle under various static preloading conditions at two frequencies are shown in figure 8 . These curves show that the present linearization method accounts for the effect of the non-linear preloading conditions on the dynamic behavior. The global response obtained with the 
finite strain model, the present model and the "linearized model without rotation" is given in figures 9(a) and 9(b). The results are very similar in each case, although those obtained with the last model mentioned (without rotation) seem to be softer than with the two other models.

\section{Concluding Comments}

Since the study by Lianis [4], situations involving the superposition of a small dynamic strain state on a large strain state have been addressed by several authors. One question was still opened: is the hypothesis that the dynamic configuration is closed to the static one is still appropriate when the geometrical or material rotations can not be neglected ? To answer this question a linearization procedure is developed, based on the polar decomposition of the dynamic strain. Only the pure deformation is assumed here to be small, and the formulation adopted is based on an expression of the internal work in which only the stress has to be linearized. In this context, it is established that linearization does not give an additive decomposition of the first Piola Kirchoff stress tensor into dynamic and static parts. The comparative tests between the present model, a finite strain model and a previous model, show that the present model is accurate and that it extends the range of validity of the existing linearized model. The use of the approach presented in this paper is obviously not restricted to a particular constitutive model, and it can be applied to other models.

\section{References}

[1] L. Mullins, Effect of stretching on the properties of rubber, Journal of Rubber Research 16 (1947) $275-289$.

[2] J. Harwood, L. Mullins, A. Payne, Stress softening in rubbers: a review, Journal of the IRI (1967) 12-27.

[3] W. Fletcher, A. Gent, Non-linearity in the dynamic properties of vulcanised rubber compounds, IRI Transaction (1953) 266-280.

[4] G. Lianis, Small deformations superposed on an initial large deformation in viscoelastic bodies, in: Procedings of the Fourth International Congress on Rheology, 1965, pp. 104-119.

[5] K. N. Morman, J. G. Nagtegaal, Finite element analysis of sinusoidal small-amplitude vibrations in deformed viscoelastic solids. part i: Theoritical development, International Journal for Numerical Methods in Engineering 19 (1983) 1079-1103.

[6] B. K. Kim, S. K. Youn, A viscoelastic constitutive model of rubber under small oscillatory load superimposed on large static deformation, Archive of Applied Mechanics 71 (2001) 748-763.

[7] B. K. Kim, S. K. Youn, W. S. Lee, A constitutive model and fea of rubber under small oscillatory load superimposed on large static deformation, Archive of Applied Mechanics 73 (2004) 781-798.

[8] P. Höfer, A. Lion, Modelling of frequency- and amplityde-dependant material properties of filler-reinforced rubber, Journal of Mechanics and Physics of Solids 57 (2009) 500-520.

[9] A. Lion, J. Retka, M. Rendek, On the calculation of predeformation-dependent dynamic modulus tensors in finite nonlinear viscoelasticity, Mechanics Research Communications 36 (2009) 653-658.

[10] G. Lesieutre, Characterization and modeling of the low strain amplitude and frequency dependent behavior of elastomeric damper material, Journal of the American helicopter society 45 (2000) 34-42.

[11] W. Luo, X. Hu, C. Wang, Q. Li, Frequency- and strain-amplitude-dependent dynamical mechanical properties and hysteresis loss of cb-filled vulcanized natural rubber, International Journal of Mechanical Sciences 52 (2010) 168-174.

[12] R. Flory, Thermodynamic relations for highly elastic materials, Transactions of the Faraday Society 57 (1961) $829-838$.

[13] J. Moreau, Sur les lois de frottement, de plasticité et de viscosité, Comptes rendus de l'Académie des sciences 271 (1970) 608-611.

[14] B. Halphen, Q. Nguyen, Sur les matériaux standards généralisés, Journal de Mécanique 1 (1975) 39-63.

[15] M. Rüter, E. Stein, Analysis, finite element computation and error estimation in transversly isotropic nearly incompressible finite elasticity, Comput. Methods Appl. Mech. Engrg. 190 (2000) 519-541.

[16] G. Holzapfel, Nonlinear Solid Mechanics, Wiley, 2004.

[17] S. Lejeunes, Modeling of laminated rubber-like/metal structures with a numerical reduction method, Ph.D. thesis, University of Aix-Marseille II (2006).

URL http://tel.archives-ouvertes.fr/tel-00090600/fr/

[18] S. Lejeunes, S. Méo, A. Boukamel, A direct numerical integration scheme for visco-hyperlastic models using radial return relaxation, European Journal of Computational Mechanics 19 (2010) 129-140.

[19] S. Lejeunes, A. Boukamel, S. Méo, Finite element implementation of nearly-incompressible rheological models based on multiplicative decompositions, Computers \& Structures 89 (2011) 411-421.

[20] R. Foerch, J. Besson, G. Cailletaud, P. Pivlin, Polymorphic constitutive equations in finite element codes, Comput. Methods Appl. Mech. Engrg. 141 (1996) 355-372.

[21] I. Babuška, R. Narasimhan, The babuška-brezzi condition and the patch test: an example, Comput. Methods Appl. Mech. Engrg. 140 (1997) $183-199$.

[22] I. Babuška, J. T. Ogden, Verification and validation in computational engineering and science: basic concepts, Comput. Methods Appl. Mech. Engrg. 193 (2004) 4057-4066. 